\title{
USER-CENTRED DESIGN FOR A NOT STRAIGHTFORWARD UNIVERSITY WAYFINDING
}

\author{
Javier PICAZO ${ }^{1}$, Amber HAZENOOT ${ }^{1}$, Celia OTADUY ${ }^{1}$, Marie BRAUX ${ }^{1}$ and Way BONG ${ }^{2}$ \\ ${ }^{1}$ European Project Semester, OsloMet - Oslo Metropolitan University, Oslo, Norway \\ ${ }^{2}$ Department of Computer Science, Faculty of Technology, Art and Science, OsloMet - Oslo \\ Metropolitan University, Oslo, Norway
}

\begin{abstract}
OsloMet - Oslo Metropolitan University receives thousands of students and visitors annually. Its main campus consists of many buildings in which students, staff and visitors navigate. Unfortunately, navigating around the campus can be challenging, as the existing wayfinding system is complex and not straightforward. This paper presents a problem-based approach to address the wayfinding challenges around the campus. A group of European Project Semester students followed a user-centred design approach to involve participants throughout the four main phases of the study-understanding, envisionment, design and evaluation. Interviews and scenario-based user testing were conducted to identify the underlying problems. The findings indicated that the numbering system for rooms was inconsistent, and the signage was not clear, visible and coherent for all the buildings on the campus. Using graphic design principles and wayfinding guidelines, a new consistent room numbering, a signage system and a mobile navigation app were proposed, developed and evaluated. The results showed that the new wayfinding system was clear and easy to understand, and it can be applied in all buildings. We observed a shorter time spent navigating to a specific room, and no mistakes was made. The app was found to be a useful and helpful tool for wayfinding. As a result of this study, the authors highlight the importance of involving users throughout the entire research process, which is our most significant learning experience as a group.
\end{abstract}

Keywords: Wayfinding system, navigation, signage system, user-centred design

\section{INTRODUCTION}

Every year, large numbers of new students and visitors come to OsloMet - Oslo Metropolitan University. At the main campus, located in the Oslo, Norway, city centre at Pilestredet, students and staff need to navigate around several buildings where faculties, libraries and other services can be found. For instance, new students need to deal with administrative matters at one building and attend classes at other buildings. Visitors who are attending meetings, conferences and other events also need to find their way around the campus. Unfortunately, navigating around the campus can be challenging, as the existing wayfinding system is complex and not straightforward. Buildings on the main campus are very different from one another in their structure, as most of them were office buildings before they became part of the university. Physical signs, information panels and other elements in the existing wayfinding system were found to be unhelpful.

This paper presents a problem-based approach to address the wayfinding challenges on the campus. Four European Project Semester (EPS) students approached this project as a user-centred design education study. By adopting a user-centred design approach, the group involved participants in the four main phases of the work - understanding, envisionment, design and evaluation.

This paper is organized as follows. After the introduction in Section 1, Section 2 of this paper provides a review of previous studies related to using user-centred design in wayfinding and navigating systems. In Section 3, we describe our research process and findings that address the wayfinding challenges around the campus. Proposed solutions, i.e. a new consistent room numbering system, a signage system and a mobile navigation app are presented in this section. In Section 4, we reflect on the learning experience during this process of design education and state the limitations of our work. 


\section{USER-CENTERED DESIGN}

According to Karat [1], user-centred design defines an iterative process whose goal is the development of usable systems. User-centred design has been applied in developing wayfinding and navigation for users with special needs, such as cognitive impairments [2] and visual impaired [3]. Chang et al. [2] developed a personal guidance system for cognitively impaired individuals to help them safely and effectively navigate by themselves, while Loomis et al. [3] argued that using user-centred design would lead to designing the most effective wayfinding devices for visually impaired travellers. Other studies adopted user-centred design in developing maps [4] and virtual reality for wayfinding in built environments [5]. However, none of these studies has demonstrated the process of engaging and involving the users throughout the research. In addition, to the best of our knowledge, students had not previously been engaged or involved in any user-centred wayfinding research.

Studies have shown that user-centred design practitioners can gather and utilize valuable input and ensure end users will be able to use the end product [6-8]. For these reasons, a user-centred design approach was suitable in this design education study, as existing wayfinding and navigating systems were found to be complicated, unhelpful and user-unfriendly to the end users. By applying a user-centred design approach, we aimed to involve the users in every phase of the study.

The research process consists of four phases, i.e. understanding, envisionment, design and evaluation. These phases were derived by referring to Benyon [9]'s design process of human-centred interactive systems. According to Benyon [9], these four activities can help designers dealing with the 'messy' problems of designing interactive systems. These phases are therefore suitable for this problem-based study since the challenges of wayfinding were complicated, and the underlying problems were not identified when the group started the project.

\section{UNDERSTANDING}

At this phase, designers have to be clear about the requirements of the system, so they are required to research users, their activities, and contexts relevant to the domain [9]. Using this approach, the underlying problems in this design education study were investigated.

\subsection{Interview}

Interviews were first conducted in the understanding phase. The aim was to explore the users' perspectives on the current wayfinding situation at OsloMet Pilestredet campus [10]. Nine students were interviewed, representing different ages (19-33 years old), nationalities (Norway, United States, Netherlands, Nigeria, Bosnia and Serbia), time spent studying at OsloMet (from first semester to second year) and programmes of study, as well as the buildings they usually visited. A semi-structured interview guide was used, with questions including, What do you think about navigating around the campus? Do you think it is easy to find a room given the room number? How you do usually find your way when you are lost? What do you think can be done to improve the wayfinding system at the campus?

From the interview results, we concluded that all the participants had problems finding their way in their first few weeks at the campus. The students usually spent additional time at same buildings where they attended lectures, and therefore, after spending some time at those buildings, they knew how the wayfinding system in some buildings worked. If they needed to go to a building they rarely visited, however, they would still have a problem finding their way. In addition to the wayfinding system, related issues brought up by the interviewees included difficulty in making reservations for rooms for their meetings, group study, self-study and etc., and checking the availability of lecturers and other staff on the campus.

\subsection{Scenario-based user testing}

In addition to the interviews, four students participated scenario-based user testing conducted in this phase. Scenario-based user testing is suitable for studying a person's behaviour to find out what he or she would do in a given situation [11]. It could therefore contribute to our goal of investigating how the users navigated around the campus and how they found their way when they got lost.

In this study's scenario-based user testing, the participants were asked to find specific rooms and places around the campus after they were given the room and building numbers, for instance, P35 PI760, P52 F318 and P48 S264, where P35, P52 and P48 are building numbers, and PI760, F318 and S264 are room numbers. The participants could use any resources they could find and seek help from others to find their way. No assistance was provided by the research group during the testing, as we aimed to simulate 
the scenario as realistically as possible. The participants were observed while performing the testing tasks, and the researchers took notes on their observations. After completing all the tasks, the participants were asked a few questions to clarify their behaviour and actions.

The findings indicated that the students managed to find the buildings, but they usually got lost when navigating inside a building. The main problem identified was the inconsistent room numbering system. A campus building is named for the street where it is located; for instance, P35 means Pilestredet 35, and PP means Pilestredet Park 33. Many foreign students had problems understanding this at first due to the language and cultural differences. However, they did not take long to understand the naming of the buildings. The challenge students struggled with the most was the naming of the various wings in different buildings. For instance, one participant commented, "In fact, I've never understood it. Maybe the buildings' wings are named in alphabetical order, because at P52, they are from A to F, and at P48 from $\mathrm{R}$ to $\mathrm{S}$. Maybe there is a relation there".

Although there were signs inside the buildings to help the students navigate, the participants did not rely much on them, as they were not visible enough. Participants who conducted the testing tasks in several buildings commented that the signs were not coherent and consistent for all buildings. This made it more difficult for the students to be aware of the signs and to understand them. Lastly, when the participants were trying to find their way, they usually relied on the navigation app.

\section{ENVISIONMENT}

Benyon [9] defines envisionment as finding appropriate media in which to render design ideas. In this phase, the findings from the understanding phase were translated into user needs. Techniques such as sketches and mood boards [9] guided the envisionment phase, with user needs and personas in mind. The outcomes of this phase are the elements of the proposed new wayfinding system:

1. A new, more intuitive and coherent room numbering system in all buildings on the campus.

2. A new physical signage system, which defines the signs' graphic characteristics as well as their optimal placement. These signs will identify the buildings on the campus by displaying the OsloMet brand to promote cohesion. For displaying room numbers, the proposed new numbering system are colour coded.

3. A mobile app that not only enhances the wayfinding experience, but also features improvements in the room-booking system and in finding out the availability of lecturers and other staff on the campus.

\subsection{Room numbering system}

The proposed room numbering system is shown in Figure 1.

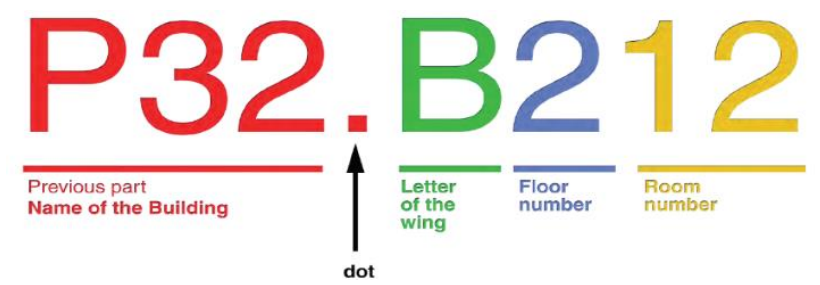

Figure 1. Proposed colour-coded room numbering system

This numbering system reflects the study results, as follows:

- Buildings' names would not change, as both the interviews and scenario-based user testing showed that the existing naming of the buildings was clear and understandable to students.

- Building wings are represented by alphabetical letters, as was already the case in most of the buildings in the campus, but participants found the current system insufficiently unified and not intuitive enough. To address this issue, we suggest the letters naming the wings follow alphabetical order so that, when entering a building, wing A would be the leftmost, and the letters would be assigned alphabetically and clockwise to consecutive wings from left to right.

- $\quad$ Floor numbers would remain the same.

- The room numbering rule was inspired by the house numbering on the street. The room numbers would flow in ascending order from one end of the building to the other, starting from the main entrance. Even numbers are on the left, and odd numbers on the right.

- Colour-coded room numbering would be used for the physical signs. 


\subsection{Physical signs}

While envisioning the physical signs, graphic design principles and guidelines were applied. One of the main design principles was the pyramid method, which explains the relationships between the three different elements that form wayfinding, i.e. information, graphics and hardware [12]. For each element, we applied design guidelines with users in mind. For instance, in terms of typography (graphics element), sans serif fonts were chosen due to the legibility, versatility and longevity of the style [13]. The placement of the signs (information element) was designed to ensure users could easily notice the signs.

\subsection{Mobile navigation app}

The main functionality of the app was to help the users find their way on the campus. However, the feedback from the interviews and scenario-based user testing indicated that the users wanted additional functionalities, such as booking a room and finding a person. The app was therefore designed so that upon opening the app, the main page displayed a map showing the user's location. This page contained a search bar and a menu icon. By default, the search bar allowed the users to enter the name of the place they wanted to find. Tapping on the menu allowed them to change to other options, such as 'find a person' and 'book a room'. Figure 2 below illustrates the menu page (left), the main page (middle) and the search result page (right).

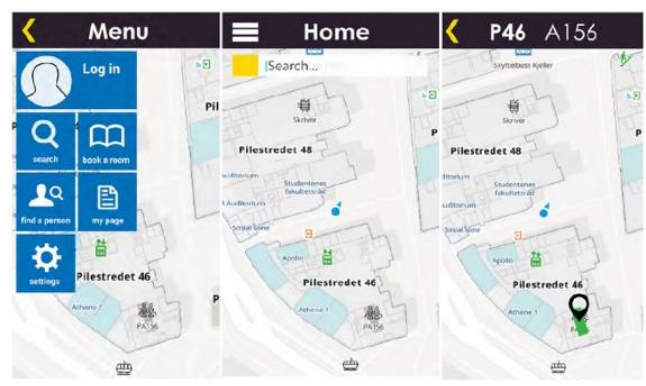

Figure 2. Mobile navigation app

\section{DESIGN}

In this phase, we engaged mostly on the physical design and the users' interactions with the prototypes Ideas from sketches and the mood boards were translated into the prototype, with the focus on the users' feel and touch. As shown in Figure 3, in terms of the room numbering system and physical signs, actualsize physical signs were created to ensure that the prototypes were as real as possible for the users' evaluation. For the mobile navigation app, a high-fidelity prototype was produced which was run using the InVision app. The prototype enabled the users to navigate from one page to another on the app.
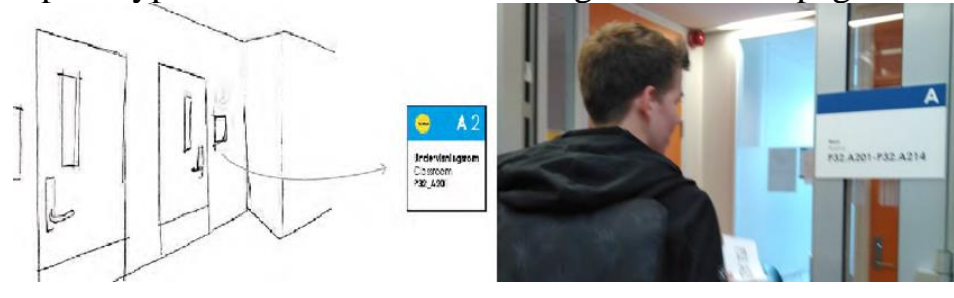

Figure 3. Translating the sketch of a physical sign for a room (left) to an actual-size physical sign (right), which was used during user testing

\section{EVALUATION}

In this phase, two types of user testing were conducted. Due to time and resource constraints, the mobile navigation app could not be included in the scenario-based user testing. The app was tested separately in the usability testing.

\subsection{Scenario-based user testing}

Five students were recruited to participate in the scenario-based user testing. The aim of scenario-based user testing in this phase was different from the understanding phase: Instead of investigating how the users navigated around the campus and how they found their way when they got lost, we wanted to evaluate how the wayfinding system worked as a whole with the proposed room numbering system and physical sign system. New physical signs and the new room numbering system were installed 
temporarily during the scenario-based user testing (see Figures 3 and 4). The participants were given testing tasks to perform, and they were observed while performing these tasks. Interviews were conducted after the testing.

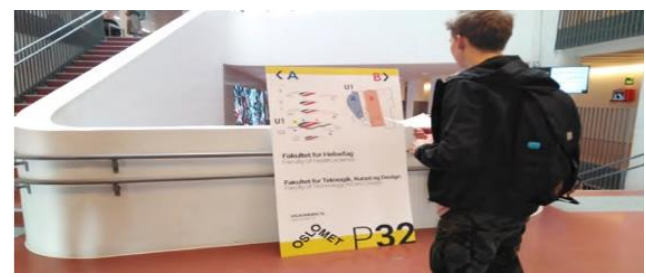

Figure 4. One of the physical signs during user testing

The user testing showed that the new wayfinding system was clear and standardized and could be applicable in all buildings. The participants commented that the proposed system was more efficient and navigating around campus with the new numbering and signage system was easier. They said the placement of the signs was better, and they could easily understand the signs and room numbers. Shorter time in navigating to the appointed room was observed, and no mistakes were made.

\subsection{Usability testing}

Following user testing guidelines [11, 14], six students evaluated the app as a high-fidelity prototype. They were asked for their opinions about each page of the app to determine if they missed something, expected something different or had any comment. All of them appreciated the design and said they would recommend that it be further developed as an actual app with a GPS tracker. The user interface and functionalities of the app were described as useful and easy to use. One important suggestion was an option to designate some rooms as favourites in the app, so users could check the availability of those rooms and book them more easily.

\section{CONCLUSIONS}

To the best of our knowledge, this design education study was the first to adopt user-centred design in addressing wayfinding challenges with students. This study demonstrated the importance of involving users throughout the entire research process in a problem-based wayfinding project. By involving users in the understanding, envisionment, design and evaluation phases, the results enabled us to propose solutions that address the underlying problems of navigating and wayfinding around the campus. The proposed solutions include a new, consistent room numbering system, a signage system and a mobile navigation app.

\subsection{Implications}

None of the EPS group members had prior knowledge of designing a wayfinding system. Similar to the findings by Wallisch et al. [6], using a user-centred design approach also served to educate and train the researchers as novice designers in this design education study. Involving users and considering user needs were proven to be crucial, as both contributed significantly to developing wayfinding solutions that were intuitive and easy for users to learn and use. Our work also demonstrates the use of usercentred design complementing design principles, which is relevant to the design education activity.

Semi-structured interviews and scenario-based user testing contributed to a large extent to identifying problems with the current wayfinding. Interviewing people was new to the research group, and it was crucial to ask questions that prompted the interviewees' feedback and gain as many insights as possible [10]. The outcomes from the interviews and scenario-based user testing identified the users' needs and requirements, which were then translated into the envisionment and design of the proposed solutions. While generalizing user needs and requirements, we applied graphic design principles and wayfinding guidelines. In the evaluation phase, user testing was conducted with users to evaluate the proposed solutions and gain feedback about them.

According to Mao et al. [8], there are lack of some common characteristics of an ideal user-centred design process, such as user experience, end-to-end user involvement in the development process and tracking customer satisfaction. Combining Benyon [9]'s design process of human-centred interactive systems with a user-centred design approach, we were able to ensure the inclusion of the abovementioned characteristics. In the user testing, our participants commented that their opinions were heard and considered. Our proposed solutions reflect the outcome of end-to-end user involvement in the whole 
research process, which also show that user-centred design approach contributed to designing end products that really meet the requirements of the users.

\subsection{Limitations and future work}

We acknowledge that there are a few limitations to our project. First, the project itself was conducted in only one semester. Due to restricted time and resources, we were able to conduct the four phases of usercentred design activities with students only, and the processes were not iterative enough. Other wayfinding users around the campus, such as staff and visitors, should be included in future studies, and the activities in each phase should be repeated with them in future iterations.

Another limitation is the use of a colour-coded room numbering system. While colour-coding the physical signs can help many users, this approach excludes colour-blind and other visual impaired users. In the future, we hope to investigate whether the use of highly contrasted colours for colour-coded room numbering would be helpful for this group of users.

Lastly, the universal design principles should be applied in future studies to ensure users with different needs are included. The campus wayfinding system is used by people with diverse abilities, preferences, knowledge, and so forth. Thus, we wish to involve users with visual, cognitive and/or other difficulties, as well as users representing different age groups in the future, to ensure the system may be used and understood to the greatest possible extent.

\section{REFERENCES}

[1] Karat, J. Evolving the scope of user-centered design. Communications of the ACM, 1997, 40(7), 33-38.

[2] Chang, Y.-J., Tsai, S.-K., Chang, Y.-S., \& Wang, T.-Y. A novel wayfinding system based on geo-coded QR codes for individuals with cognitive impairments. In Proceedings of the 9th international ACM SIGACCESS conference on Computers and accessibility, Tempe, October 2007, pp.231-232 (Association for Computing Machinery, New York)

[3] Loomis, J. M., Golledge, R. G., Klatzky, R. L., \& Marston, J. R. Assisting Wayfinding in Visually Impaired Travelers. Applied spatial cognition: From research to cognitive technology, 2007, 179-202, (Lawrence Erlbaum Associates Publishers).

[4] Porathe, T. User-centered map design. In Usability professionals' association conference, Austin, June 2007.

[5] Vilar, E. and Rebelo, F. Virtual reality in wayfinding studies. In International Conference on Applied Human Factors and Ergonomics, Las Vegas, July 2008.

[6] Wallisch, A., Pérez-Villalobos,C., Briede-Westermeyer, J.C. \& Paetzold, K. Bridging the gap- A co-creation experience of novice designers and elderly users. In E\&PDE, Glasgow, September 2019.

[7] Abras, C., Maloney-Krichmar, D., and Preece, J. User-centered design. Berkshire Encyclopedia of Human-Computer Interaction, 2004. 37(4), pp.445-456 (Thousand Oaks: Sage Publications).

[8] Mao, J. Y., Vredenburg, K., Smith, P. W., \& Carey, T. The state of user-centered design practice. Communications of the ACM, 2005, 48(3), 105-109.

[9] Benyon, D. Designing interactive systems: A comprehensive guide to HCI, UX and interaction design, 2014 (Pearson Education Limited).

[10] Boyce, C. and Neale, P. Conducting in-depth interviews: A guide for designing and conducting in-depth interviews for evaluation input. Monitoring and Evaluation - 2. Pathfinder International, 2003.

[11] Barnum, C.M. Usability testing essentials: ready, set... test!, 2010 (Elsevier).

[12] Calori, C. and Vanden-Eynden, D. Signage and wayfinding design: a complete guide to creating environmental graphic design systems, 2015 (Wiley Online Library).

[13] Arditi, A. and Cho, J. Serifs and font legibility. Vision research, 2005, 45(23), 2926-2933.

[14] Nielsen, J. Why you only need to test with 5 users. Available: https://www.nngroup.com/articles/why-you-only-need-to-test-with-5-users/ [Accessed on 2020, 10 February], (2000) 18 March. 